\title{
Criminologie
}

\section{Quand droit et criminologie se rencontrent}

\section{Hélène Dumont}

Volume 40, numéro 2, automne 2007

Peines et pénalité au Canada. Autour des travaux de Pierre

Landreville

URI : https://id.erudit.org/iderudit/016854ar

DOI : https://doi.org/10.7202/016854ar

Aller au sommaire du numéro

Éditeur(s)

Les Presses de l'Université de Montréal

ISSN

0316-0041 (imprimé)

1492-1367 (numérique)

Découvrir la revue

Citer cet article

Dumont, H. (2007). Quand droit et criminologie se rencontrent. Criminologie, 40(2), 103-106. https://doi.org/10.7202/016854ar d'utilisation que vous pouvez consulter en ligne.

https://apropos.erudit.org/fr/usagers/politique-dutilisation/ 


\section{Quand droit et criminologie se rencontrent}

\section{Hélène Dumont}

Professeure titulaire

Faculté de droit

Université de Montréal

helene.dumont@umontreal.ca

Il existe dans un parcours universitaire la rencontre de quelques professeurs qui influencent notre futur. Pierre Landreville a été pour moi l'un de ceux-là. Au début des années 1970, je commence des études supérieures en droit pénal à la Faculté de droit de l'Université de Montréal à un moment où celle-ci introduit un loup dans la bergerie, soit un criminologue dans une tanière de juristes. Les études en droit se suffisaient alors à elles-mêmes et n'offraient, en tout cas, aucune initiation à la criminologie aux étudiants intéressés par le droit pénal, soit pour le pratiquer, soit pour en faire un champ d'études. Il y avait à cette époque une frontière intellectuelle, voire un mur de Berlin, entre le droit et les sciences sociales en général et entre le droit pénal et la criminologie en particulier.

Pierre Landreville enseigne ainsi à un petit groupe de mordus du droit pénal les rudiments de la criminologie et de la pénologie. Ces derniers étaient des «fans» du pénaliste Jacques Fortin, le professeur qui avait planifié l'entrée de la criminologie dans un cursus de maîtrise en droit pénal. Je me rappelle avoir fait une recherche sur les règles minimales concernant le traitement des détenus ${ }^{1}$, un travail servant

1. Ensemble des règles minima pour le traitement des détenus, Résolution du premier congrès des Nations Unies pour la prévention du crime et le traitement des délinquants, Genève, 30 août 1955. 
d'évaluation dans le cadre du cours de Pierre et qui sera par la suite l'aiguillon de mes premières pistes de recherche en tant que professeure de droit pénal. Dès lors, je m'intéresse aux droits des détenus et pas seulement de manière académique. Pierre Landreville me gagne en effet à son militantisme en faveur de l'amélioration des conditions de détention dans les prisons provinciales et dans les pénitenciers fédéraux. Nous sommes du nombre des activistes de la première heure de l'Office des droits des détenu(e)s au sein de la Ligue des droits de l'Homme ${ }^{2}$. C'est d'ailleurs à l'intérieur de cet organisme que nous réclamons la fermeture de Parthenais ${ }^{3}$, un centre de détention provisoire des prévenus logé au-dessus des locaux de la police et manifestant, dans son organisation et son architecture, une totale indifférence à l'égard de leurs droits selon les prescriptions de l'ONU en cette matière. Dans la foulée de ce militantisme, nos recherches communes tenteront de vérifier la conformité ou la non-conformité des directives du commissaire aux pénitenciers canadiens avec les règles onusiennes sur le traitement des détenus ${ }^{4}$. Nous avions un obstacle de taille à cet exercice intellectuel puisque les directives étaient secrètes et échappaient à l'obligation de publicité des textes réglementaires ${ }^{5}$. En somme, toute la matière du droit carcéral était inaccessible et le droit correctionnel n'existait tout simplement pas. Intenter un recours devant un tribunal compétent au motif de la violation des droits d'un détenu relevait de l'aventure «kafkaesque». D'ailleurs la Cour suprême sanctionnera vers la fin des années 1970 par une fin de non-recevoir la requête d'un détenu contestant la légalité d'une décision disciplinaire affectant ses droits sous prétexte qu'il s'était présenté devant le mauvais tribunal de première instance, que les directives régissant les conditions de détention fédérales n'étaient pas des règlements et que les droits des détenus étaient assimilables à des privilèges. Ce jugement retardera de plusieurs années la reconnaissance ultérieure par cette Cour des droits fondamentaux des détenus ${ }^{6}$. En

2. J'ai été présidente de l'Office des droits des détenu(e)s de la Ligue des droits de l'Homme du Québec (1974-1975) après Pierre Landreville.

3. Dumont \& Gagnon (1976). Parthenais, une lutte à finir. Criminologie, IX (1-2), 163189.

4. Landreville, P., \& Dumont, H. (1973). Discipline et droits des détenus dans les institutions pénales au Québec. Revue canadienne de criminologie/Canadian Journal of Criminology, $15,412-435$.

5. Martineau c. Comité de discipline des détenus de l'Institution de Matsqui, [1978] 1 RCS 118.

6. Pour une étude complète de l'évolution de la jurisprudence en droit carcéral, voir les travaux de Lucie Lemonde, qui a fait sa thèse de doctorat sous la supervision conjointe 
ressassant pour l'occasion mes souvenirs, je suis convaincue que les travaux scientifiques et l'action engagée de Pierre Landreville ne sont pas étrangers à l'évolution de la politique pénale en matière de droits des détenus au Québec et au Canada.

Depuis nos premiers échanges sur la question des droits des détenus, notre production scientifique respective s'est effectuée en citant avec approbation les travaux de l'un et de l'autre et en enrichissant nos propres recherches, de criminologie pour ma part et de droit pour sa part. Il me faut à cet égard témoigner de son influence dans la façon de présenter mon Traité de pénologie 7 aux juristes canadiens. En 1993, je publie un traité sur les règles de détermination et d'exécution des sentences au Canada en cherchant à expliquer non seulement le droit tel qu'il s'élabore dans la loi et par la jurisprudence, mais en examinant aussi les courants de pensée qui surdéterminent ce droit de manière systémique et en intégrant les études empiriques des criminologues à la compréhension de la peine sur le plan juridique. En bref, il me paraissait inconcevable de faire de la pénologie sans l'apport des études émanant de la criminologie et cette conviction, je l'ai acquise au contact de Pierre Landreville, le professeur, le collègue, l'humaniste et le scientifique.

En lisant le bilan que trace Pierre Landreville dans ce numéro de Criminologie et qu'il intitule «Grandeurs et misères de la politique pénale au Canada: du réformisme au populisme», je trouve encore matière à l'évolution de ma propre réflexion. En effet, Pierre fait état du décalage qui existerait entre, d'une part, le discours politique conservateur actuel en faveur de plus de sécurité et de rigueur répressive et, d'autre part, la pratique des intervenants dans le domaine pénal et correctionnel. En d'autres mots, les pratiques professionnelles au Canada ne suivraient pas nécessairement de façon concomitante le discours populiste ambiant et resteraient influencées par les connaissances des sciences humaines et sociales et contribueraient peut-être à modérer les effets des peines plus rigoureuses adoptées ces récentes années. J'aime à penser que les professionnels formés aux sciences humaines et sociales et les juristes

de Pierre Landreville et d'Hélène Dumont et qui publie dans cet ouvrage «Landreville et les détenu(e)s de par ici». Voir aussi Lemonde \& Landreville (2002). La reconnaissance des droits fondamentaux des personnes incarcérées: l'expérience canadienne. In Kaminski \& De Schutte (Dir.), L'institution du droit pénitentiaire. Enjeux de la reconnaissance des droits des détenus (69-88), Paris: Bruylant, LGDJ.

7. Dumont, H. (1993). Pénologie: le droit canadien relatif aux peines et aux sentences. Montréal: Les Éditions Thémis Inc. 
initiés à la criminologie comme je l'ai été, grâce à Pierre Landreville, réussiront à mieux résister à ce mouvement répressif et à imprégner la justice de valeurs humanistes favorables à la réinsertion sociale des détenus. Pierre Landreville y a travaillé tout au long de sa carrière et il m'a inspirée depuis le commencement de la mienne.

Larticle de Pierre dans ce numéro de la revue Criminologie confirme à nouveau la pertinence de ces transferts de connaissances entre le droit pénal et la criminologie. Aujourd'hui, l'on appellerait plus sérieusement ces échanges formels et informels entre Pierre et moi, marqués du sceau de l'amitié tout au long de nos carrières universitaires, de l'interdisciplinarité. 